\title{
Autologous Ovarian Cancer-specific Cytotoxic T-Lymphocytes
}

National Cancer Institute

\section{Source}

National Cancer Institute. Autologous Ovarian Cancer-specific Cytotoxic T-Lymphocytes. NCI Thesaurus. Code C155664.

A preparation of autologous cytotoxic T-lymphocytes (CT Ls) genetically modified to target a not yet disclosed ovarian cancer-specific tumor-associated antigen (TAA), with potential immunomodulating and antineoplastic activities. After isolation, transduction, expansion in culture, and reintroduction into the patient, the autologous ovarian cancerspecific cytotoxic T-lymphocytes (OC-CTLS) bind to and induce selective toxicity in tumor cells expressing the TAA. 